\title{
Association between serum TSH levels and metabolic components in euthyroid subjects: a nationwide population-based study
}

This article was published in the following Dove Press journal:

Diabetes, Metabolic Syndrome and Obesity: Targets and Therapy

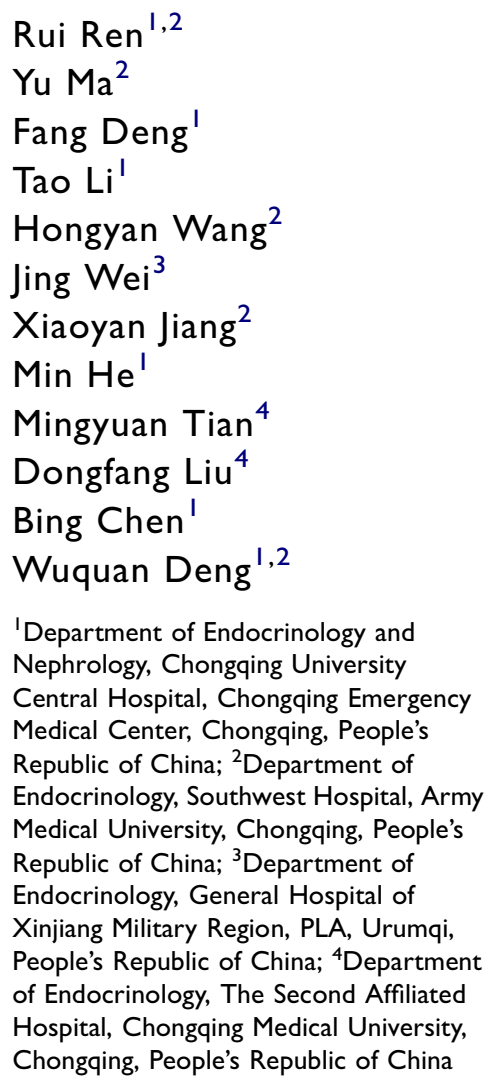

Background: Whether a relationship exists between various metabolic factors and thyroidstimulating hormone (TSH) levels in euthyroid persons remains unknown. This study aims to explore the relationship between TSH levels and metabolic factors in euthyroid individuals. Methods: A total of 2,663 subjects were enrolled from a nationwide population-based crosssectional survey of iodine nutrition, thyroid disease and diabetes in China (2014-2017). Euthyroid individuals were divided into four groups according to quartiles of TSH levels: group A ( $n=305$, 0.3-1.3 mIU/L), group B ( $\mathrm{n}=829,1.3-2.2 \mathrm{mIU} / \mathrm{L})$, group $\mathrm{C}(\mathrm{n}=673,2.2-3.2 \mathrm{mIU} / \mathrm{L})$ and group $\mathrm{D}$ $(\mathrm{n}=349,3.2-4.2 \mathrm{mIU} / \mathrm{L})$. Anthropometric parameters, biochemical indicators and TSH levels were determined.

Results: A total of 2,156 euthyroid subjects with serum TSH levels within the normal range accounted for $86.8 \%$ of the sample. The systolic blood pressure (SBP) in group D was significantly higher than that in the other three groups. Group C displayed significantly lower thyroid peroxidase antibody (TPOAb) levels than the other three groups. Group $\mathrm{C}$ also had lower anti-thyroglobulin antibody (TgAb) levels than groups $\mathrm{A}$ and $\mathrm{D}$, whereas the $\mathrm{TgAb}$ levels in group B were only lower than those in group A. Spearman's or Pearson's linear regression analysis showed that $\mathrm{SBP}(\mathrm{r}=0.054 ; P=0.013)$ was positively correlated with TSH, but cholesterol (TC) $(\mathrm{r}=-0.043, P=0.047)$ was negatively correlated with TSH. Multiple stepwise regression analysis revealed that SBP, the urinary iodine concentration (UIC), waist circumference (WC), body mass index (BMI), TC, triglycerides (TGs) and low-density lipoprotein cholesterol (LDL-C) were independent predictors of serum TSH levels.

Conclusion: This large population-based study demonstrates a significant interaction between metabolic factors and TSH levels. An adverse weight status, high blood pressure levels, blood lipid metabolism disorder and excessive iodine intake may be early manifestations of thyroid disease in euthyroid subjects.

Keywords: thyroid-stimulating hormone, metabolic syndrome, iodine nutrition, euthyroid state

\section{Introduction}

Recently, the incidence of thyroid disease has sharply increased year by year. ${ }^{1}$ The proportion of individuals with elevated TSH levels in the general population is also increasing. Hypothyroidism and subclinical hypothyroidism $(\mathrm{SCH})$ are very common diseases in the general population. The reported incidence of $\mathrm{SCH}$ ranges between $5 \%$ and $10 \%$ and is higher in older people and women. ${ }^{2}$ Based on a survey of the prevalence of thyroid diseases in 2010, the prevalence of SCH in China was $16.7 \%$ based on a diagnostic cut-off level of thyroid-stimulating hormone (TSH) $>4.2 \mathrm{mIU} / \mathrm{L}$.

Correspondence: Wuquan Deng Department of Endocrinology and Nephrology, Chongqing University Central Hospital, Chongqing Emergency Medical Center, No. I Jiankang Road, Yuzhong District, Chongqing, People's

Republic of China

Tel +862363692185

Email wuquandeng@gmail.com 
High TSH levels within the normal range may be a sign of early hypothyroidism and are associated with an increased prevalence of autoimmune thyroid disease and a risk of hypothyroidism in the near future. ${ }^{3}$ An increased serum TSH level may cause multiple organ damage and metabolism disorder. ${ }^{4-8}$ Inoue et al reported that a high-normal TSH level can increase the risk of all-cause, cardiovascular and cancerrelated mortality compared with medium-normal TSH levels. ${ }^{4}$ Coronary heart disease has been reported to be associated with the lowest tertile of TSH levels in men. ${ }^{5}$ Fernándezreal-Real et al found a positive correlation between TSH and postprandial fasting insulin levels in healthy euthyroid subjects. ${ }^{6}$ Although the baseline TSH level was not associated with the risk of diabetes, after 84,595 personyears of follow-up, the TSH level was demonstrated to be an additional risk factor for the development of type 2 diabetes in euthyroid subjects. ${ }^{7}$ In early pregnancy, $\mathrm{SCH}$ and thyroperoxidase antibody-positive euthyroidism are associated with an increased risk of gestational diabetes mellitus. ${ }^{8}$ Additionally, glycemic aberrations are closely associated with TSH secretion in type 1 diabetes regardless of variations in thyroid hormone concentrations. ${ }^{9}$ However, in a previous meta-analysis, plasma TSH levels within the normal range had no significant relationship with the incidence of type 2 diabetes, even in patients with a high cardiovascular risk. ${ }^{10}$

In addition to the results of the above studies, the prevalence of metabolic syndrome in China has recently been reported to be as high as $33.9 \%$; $^{11}$ thus, disorder of the TSH level may be influenced by metabolic regulation factors. However, the results are not completely consistent in previous studies, ${ }^{4,5,7,10}$ and the underlying cause is not fully known. The possible reasons include small sample sizes, racial differences and participant selection from special populations. In addition, reports including the urinary iodine concentrations (UIC) are rare or conflicting. The relationship between iodine intake and TSH is unclear. Therefore, we performed a cross-sectional investigation based on a nationwide iodine nutrition and thyroid disease epidemiological survey to explore the relationship between TSH and thyroid antibody, the UIC, blood glucose, blood lipids, blood pressure, and other metabolic parameters in euthyroid subjects.

\section{Subjects and methods Study population}

The National Survey of Iodine Nutrition, Thyroid Diseases and Diabetes (2014-2017) is a nationwide, cross-sectional survey of the Chinese general population. Participants were randomly selected from two urban and two rural communities in a southwest city with a population of thirty million inhabitants. A total of 2,663 participants aged 18 years or older (18-96 years old) were included in this study. Among these individuals, 180 subjects were excluded due to incomplete data. Thus, 2,483 subjects remained for the study analysis. The study subjects met the following inclusion criteria: (1) 18 years of age or older; (2) Han race; (3) residence in a community (village) for more than 5 years; (4) no iodine contrast agent examination or amiodarone intake in the past three months; and (5) nonpregnant women. The study was approved on April 25, 2013, by the ethics committee of the First Hospital of China Medical University (No. 2013-115), and written informed consent was obtained from all participants.

\section{Anthropometric and biochemical measurements \\ Anthropometric parameters}

All of the participants were required to fast overnight before the physical examination. The anthropometric parameters included height, weight, waist circumference (WC) and blood pressure. Height, weight, waist circumference and blood pressure were measured according to standard procedures reported in a previous study. ${ }^{12}$ The participants should rest for 10 mins before blood pressure measurement, relax their minds, avoid exertion, empty the bladder, stop smoking 15 mins before the test, avoid drinking tea and coffee, and be seated. The right upper limb blood pressure was measured twice per person for 3-5 mins each time.

\section{Experimental procedures and determination methods}

Overnight fasting blood samples were collected. All subjects were assayed for $\mathrm{TSH}$, thyroid peroxidase antibody (TPOAb), anti-thyroglobulin antibody (TgAb), total cholesterol (TC), triglycerides (TGs), low-density lipoprotein cholesterol (LDL-C), high-density lipoprotein cholesterol (HDL-C), blood uric acid (UA), fasting blood glucose (FBG), glycosylated hemoglobin (HbAlc), and the UIC. ${ }^{12,13}$ The TSH, TPOAb and TgAb levels in the specimens were determined by immunochemiluminescence. The FBG level was assayed using the glucose oxidase method. The TC, TG, LDL-C, HDL-C and UA concentrations were determined enzymatically. The HbA1c level was determined using high-performance liquid chromatography. The UIC was uniformly determined with inductively coupled plasma 
mass spectrometry (ICP-MS) (Agilent 7700×, Agilent Technologies, USA) in the central laboratory.

\section{Definitions of thyroid function and the normal reference ranges}

The subjects with normal thyroid function were divided into four groups according to quartiles of TSH levels: group A $(\mathrm{n}=305,0.3-1.3 \mathrm{mIU} / \mathrm{L})$, group $\mathrm{B}(\mathrm{n}=829$, $1.3-2.2 \mathrm{mIU} / \mathrm{L})$, group $\mathrm{C}(\mathrm{n}=673,2.2-3.2 \mathrm{mIU} / \mathrm{L})$, and group $\mathrm{D}(\mathrm{n}=349,3.2-4.2 \mathrm{mIU} / \mathrm{L})$. The reference ranges were $0.3-4.2 \mathrm{mIU} / \mathrm{L}$ for $\mathrm{TSH}, 0-34 \mathrm{IU} / \mathrm{mL}$ for $\mathrm{TPoAb}$, and $0-115 \mathrm{IU} / \mathrm{mL}$ for $\mathrm{TgAb}$ as provided by the central laboratory. When the serum TSH and FT4 levels were within the normal ranges, the subject was considered to have normal thyroid function.

\section{Statistical analysis}

The experimental data were processed with SPSS statistical software, version 22.0. Continuous variables are described as the means \pm standard deviation, comparisons between multiple groups were performed with analysis of variance (ANOVA), and pairwise comparisons were performed with the least-significant difference (LSD) test. Correlations between variables were determined using Pearson's or Spearman's correlation analysis, and the independent influencing factors of TSH were explored through multiple stepwise regression analysis. A test level of $P<0.05$ was considered statistically significant.

\section{Results}

According to the analysis results of the samples, 2,156 euthyroid subjects with normal serum TSH levels remained after 327 individuals were excluded because of thyroid diseases. As presented in Table 1 and Figure 1, body mass index (BMI), WC, FBG, HbA1c, UA, TGs, TC, LDL-C and HDL-C did not differ among the groups. Comparisons of the groups showed that the systolic blood pressure (SBP) in group D was significantly higher than that in the other three groups. Group $\mathrm{C}$ displayed significantly lower TPoAb levels (Figure 1A) than those in the other three groups. Group $\mathrm{C}$ also had lower $\mathrm{TgAb}$ levels (Figure 1B) than those in group A and group D, whereas the $\mathrm{TgAb}$ levels in group B were only lower than those in group A. The difference of UIC levels between all groups were showed in Figure 1C.

As presented in Figure 2, correlations were observed between TSH levels and SBP $(\mathrm{r}=0.054 ; \quad P=0.013)$
(Figure 2A) and TC $(\mathrm{r}=-0.043, P=0.047)$ (Figure 2B). No correlations were found between the remaining metabolic indicators and TSH. Multiple stepwise regression analysis revealed that WC, BMI, SBP, TC, TGs, LDL-C and the UIC were independently related to TSH levels (Table 2).

\section{Discussion}

Thyroid disease is one of the most common endocrine system diseases. Hypothalamic-pituitary-thyroid axisrelated hormone levels are slightly altered and may affect the body overall. ${ }^{14,15}$ Increased risks of thyroid disease and type 1 diabetes have been found in children born to women with Graves' disease. ${ }^{16}$ TSH within the reference range has been reported to be positively associated with the risk of future hypothyroidism. ${ }^{3}$ The prevalence of metabolic syndrome in China is markedly high. ${ }^{11}$ Another study revealed that patients with metabolic syndrome had a $21 \%$ excess risk of developing $\mathrm{SCH}$, even after controlling for risk factors during an average follow-up of 4.2 years. ${ }^{17}$ Whether early changes in serum TSH levels are related to metabolic indicators in euthyroid subjects is unclear. In the present study, SBP, as one of the metabolic syndrome components, was higher in the highest quartiles of TSH levels. The TSH level was also positively correlated with SBP. The results are consistent with those of a previous cross-sectional meta-analysis. ${ }^{18}$ The study enrolled 2,289 euthyroid individuals and identified strong evidence for a doseresponse relationship between TSH levels and the hypertension risk. Individuals with higher normal TSH levels are at a higher risk of developing hypertension than those with lower normal TSH levels. This result was further supported by a meta-analysis suggesting a significant dose-response relationship between TSH levels and the hypertension risk in euthyroid subjects. ${ }^{19}$

In addition to influencing blood pressure, the effect of thyroid dysfunction on lipid metabolism is not fully clear. ${ }^{20}$ The thyroid is widely believed to be the target organ of lipotoxicity. ${ }^{21}$ TC and TG levels were positively correlated with TSH levels in euthyroid subjects in a previous study. ${ }^{22}$ However, the TC level was slightly negatively correlated with TSH levels in our study. Furthermore, the study found that lipid levels (TC and TG) were more likely to be abnormal in subjects with the lowest or highest quartiles of TSH levels. We speculated that a trend toward an abnormal TSH level substantially more adversely influenced blood lipid metabolism and related clinical outcomes than mediumnormal TSH levels. These findings may explain why the 
Table I Comparison of the clinical characteristics of all groups

\begin{tabular}{|c|c|c|c|c|c|c|}
\hline & $A(n=305)$ & B $(n=829)$ & $C(n=673)$ & $D(n=349)$ & $\mathbf{F}$ & $P$-value \\
\hline Age & $43.81 \pm 15.62$ & $42.13 \pm 16.89$ & $41.92 \pm 17.57$ & $43.54 \pm 19.01$ & 1.382 & 0.247 \\
\hline BMI & $23.26 \pm 3.50$ & $23.28 \pm 3.54$ & $23.44 \pm 3.63$ & $23.47 \pm 3.75$ & 0.438 & 0.726 \\
\hline SBP & $1 \mid 8(|10| 3 \mid)$, & $120(|1|, \mid 30)$ & $119(109,132)$ & $123(|||,| 36)^{a, b, c}$ & 11.323 & $0.010 *$ \\
\hline DBP & $78.19 \pm 11.83$ & $78.02 \pm 11.19$ & $78.15 \pm 11.37$ & $79.41 \pm 11.00$ & 1.336 & 0.261 \\
\hline WC & $80.64 \pm 9.91$ & $80.56 \pm 9.70$ & $80.38 \pm 10.07$ & $79.88 \pm 9.72$ & 0.457 & 0.713 \\
\hline HR & $77.34 \pm 12.01$ & $76.59 \pm 10.88$ & $76.78 \pm 10.66$ & $77.14 \pm 10.15$ & 0.450 & 0.717 \\
\hline FBG & $5.5| \pm| .58$ & $5.47 \pm 1.58$ & $5.52 \pm 1.47$ & $5.43 \pm 1.43$ & 0.326 & 0.806 \\
\hline TG & $1.54 \pm 1.33$ & $|.52 \pm| .4 \mid$ & $1.60 \pm 1.52$ & $1.59 \pm 1.31$ & 0.533 & 0.659 \\
\hline TC & $4.60 \pm 1.02$ & $4.56 \pm 0.96$ & $4.52 \pm 0.93$ & $4.49 \pm 0.91$ & 0.897 & 0.442 \\
\hline LDL-C & $2.56 \pm 0.73$ & $2.6 I \pm 0.73$ & $2.56 \pm 0.70$ & $2.55 \pm 0.69$ & 0.818 & 0.484 \\
\hline HDL-C & $1.33 \pm 0.39$ & $1.31 \pm 0.56$ & $1.29 \pm 0.39$ & $1.29 \pm 0.35$ & 0.838 & 0.473 \\
\hline UA & $345.72 \pm 98.11$ & $353.46 \pm 103.39$ & $356.57 \pm 110.39$ & $345.88 \pm 110.36$ & 1.212 & 0.304 \\
\hline $\mathrm{HbAlc}$ & $5.39 \pm 1.04$ & $5.38 \pm 1.01$ & $5.4 I \pm 0.99$ & $5.34 \pm 0.82$ & 0.424 & 0.736 \\
\hline
\end{tabular}

Notes: ${ }^{a} P<0.05$ compared with group A. ${ }^{b} P<0.05$ compared with group $B$. ${ }^{c} P<0.05$ compared with group $C$.

Abbreviations: BMI, body mass index; SBP, systolic blood pressure; DBP, diastolic blood pressure; WC, waist circumference; HR, heart rate; FBG, fasting blood glucose; TG, triglyceride; TC, total cholesterol; LDL-C, low-density lipoprotein cholesterol; HDL-C, high-density lipoprotein cholesterol; UA, uric acid; HbA Ic, glycated hemoglobin.
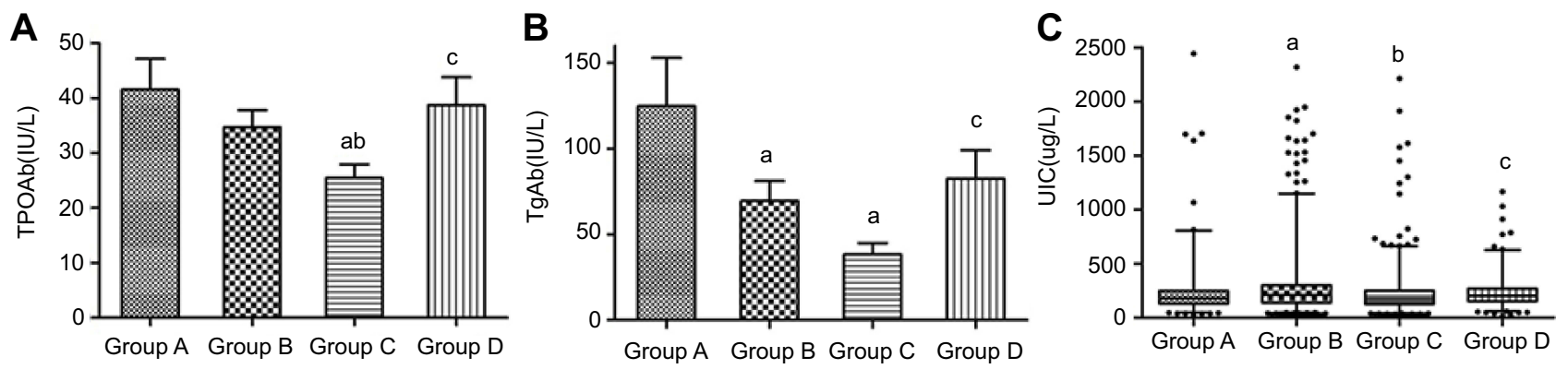

Figure I Comparison of TPOAb (A), TgAb (B), and UIC (C) levels in all groups.

Notes: ${ }^{a} P<0.05$ compared with group A. ${ }^{b} P<0.05$ compared with group $B$. ${ }^{c} P<0.05$ compared with group $C$.

Abbreviations: TPOAb, thyroid peroxidase antibody; TgAb, anti-thyroglobulin antibodies; UIC, urinary iodine.

A

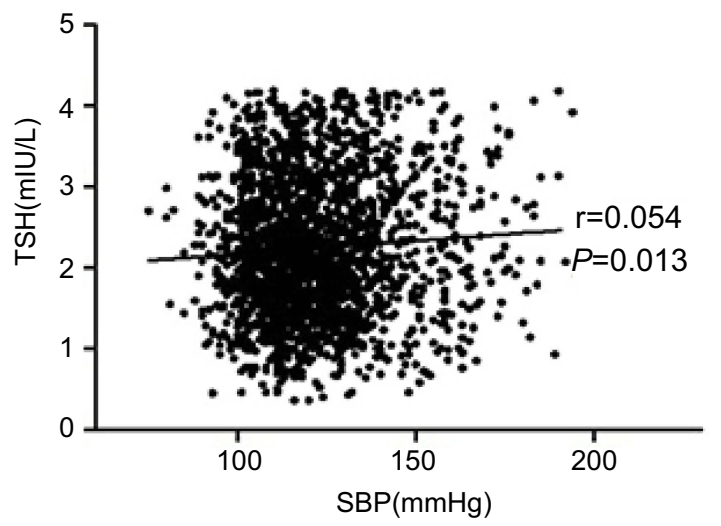

B

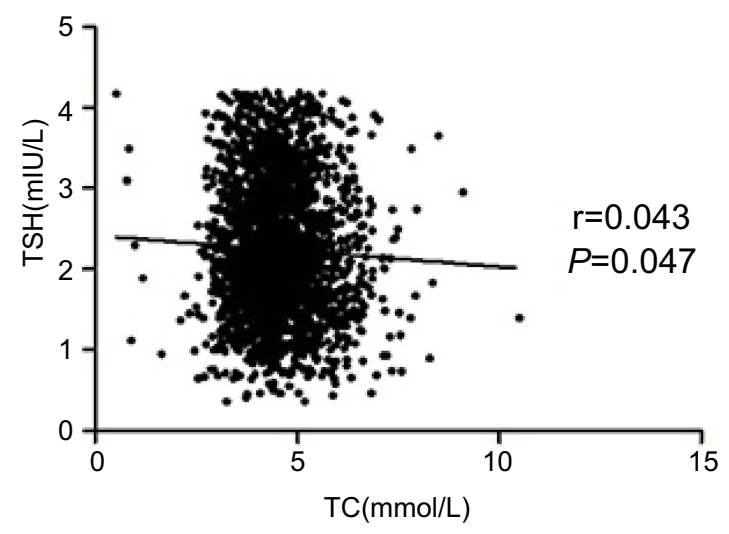

Figure 2 The correlations between TSH and SBP (A) and TC (B).

Abbreviations: TSH, thyroid-stimulating hormone; SBP, systolic blood pressure; TC, total cholesterol. 
Table 2 Multiple stepwise regression analysis of metabolic parameters and TSH levels

\begin{tabular}{|l|l|l|l|l|l|}
\hline \multirow{2}{*}{} & \multicolumn{2}{|l|}{ Nonstandardized coefficient } & Standardized coefficient & t & \multirow{2}{*}{$\boldsymbol{P}$-value } \\
\cline { 2 - 5 } & B & Standard error & & 3.956 & \\
\hline SBP & 0.004 & 0.001 & 0.093 & -3.374 & $<0.001$ \\
TC & -0.119 & 0.035 & -0.132 & -2.096 & 0.001 \\
UIC & 0.000 & 0.000 & -0.045 & -3.887 & 0.036 \\
WC & -0.011 & 0.003 & -0.132 & 2.58 & $<0.001$ \\
BMI & 0.02 & 0.008 & 0.084 & 0.01 & 2.527 \\
TG & 0.037 & 0.015 & 0.061 & 2.028 & 0.012 \\
LDL-C & 0.092 & 0.046 & 0.077 & 0.043 \\
& 2.432 & 0.186 & & 13.111 & $<0.001$ \\
\hline
\end{tabular}

Abbreviations: TSH, thyroid-stimulating hormone; SBP, systolic blood pressure; TC, total cholesterol; UIC, urinary iodine concentration; WC, waist circumference; BMI, body mass index; TG, triglyceride; LDL-C, low-density lipoprotein cholesterol.

highest or lowest normal TSH level can increase the risk of cardiovascular disease. ${ }^{4,5}$ Our hypothesis is further supported by a recent study reported by Ahi et al. ${ }^{23}$

Significantly elevated TPOAb and TgAb levels were observed in participants with the lowest and highest limits of TSH within the reference range. Thyroid-related antibody levels may be an early manifestation of thyroid diseases, especially autoimmune thyroid disease. A previous study also suggested that TPOAb and/or $\mathrm{TgAb}$ increased significantly with increasing levels of TSH and was associated with the prevalence of hypothyroidism. ${ }^{24,25}$ The UIC was highest in subjects with a TSH level of 1.3$2.2 \mathrm{mIU} / \mathrm{L}$, which is also consistent with the findings of a previous study. ${ }^{26}$ Additionally, the UIC was negatively correlated with TSH levels in euthyroid subjects. More than adequate iodine intake may be an important inducing factor of thyroid disease. The prevalence of clinical hyperthyroidism and Graves' disease has been reported to be significantly increased among Chinese populations in areas with adequate iodine intake. ${ }^{27}$

Metabolic syndrome components, weight status (BMI and WC), blood pressure, blood lipids and iodine status are all influenced by TSH levels. Adipocyte- and hepatocyte-derived metabolic regulators may be critical links between metabolic syndrome and thyroid function. ${ }^{28}$ The study results showing that WC and BMI were independently related to TSH levels are partly consistent with those of a previous Asian study revealing that men with a higher TSH concentration had higher body weights and BMI values, even after adjusting for related confounders. However, the TSH level was not associated with WC. ${ }^{29} \mathrm{~A}$ 1-mIU/L higher TSH level within the euthyroid or subclinical hypothyroid range was associated with a $0.51-\mathrm{kg}$ higher baseline weight in women. ${ }^{30}$ In patients with $\mathrm{SCH}$, high blood pressure and high serum TGs were associated with an increased risk of thyroid disease. Subjects with metabolic syndrome were reported to be at a greater risk of developing SCH. ${ }^{17}$ Thyroid hormone supplementation increased TSH levels, which reached a steady state at an incidence risk ratio of 1.75 after ten years of iodine fortification. $^{31}$

Some inevitable limitations exist in our study. First, this study was cross-sectional, and the results should be verified by a longitudinal clinical study in the future. Second, comorbidities and concomitant drug use are common among elderly individuals. According to the literature, some drugs, such as estrogen, aspirin, nonsteroidal anti-inflammatory drugs, and glucocorticoids, can affect thyroid hormones. ${ }^{32}$ We hope to provide supporting data and references for future studies, and more studies are needed to clarify the relationship between metabolic indicators and thyroid disease.

\section{Conclusion}

In conclusion, metabolic parameters showed significant changes at different levels of TSH. Weight status, blood pressure, blood lipids and iodine intake were independent predictors of serum TSH levels. Disorder of various metabolic parameters is an important factor that cannot be ignored as an influencing factor of thyroid disease. Further studies are needed to explore the possible mechanism of metabolic syndrome and its influence on thyroid function.

\section{Ethical approval and informed consent}

The study was approved on April 25, 2013, by the ethics committee of the First Hospital of China 
Medical University (No. 2013-115), and all procedures in the study were performed in accordance with the ethical standards of the 1964 Declaration of Helsinki. Written informed consent was obtained from all individual participants included in the study.

\section{Acknowledgments}

This work was funded in part by a grant awarded to Dr. Wuquan Deng from the Fundamental Research Funds for the Central Universities at Chongqing University (Grant No. 2019CDYCYB020). Additional funding was received from the Research Fund for Public Welfare of the National Health and Family Planning Commission of China (Grant No. 201402005) and the clinical research fund of the Chinese Medical Association (Grant No. 15010010589).

\section{Disclosure}

The authors report no conflicts of interest related to this work.

\section{References}

1. Bano A, Dhana K, Chaker L, et al. Association of thyroid function with life expectancy with and without cardiovascular disease: the Rotterdam study. JAMA Intern Med. 2017;177(11):1650-1657. doi:10.1001/jamainternmed.2017.4836

2. LeFevre ML; U.S. Preventive Services Task Force. Screening for thyroid dysfunction: U.S. preventive services task force recommendation statement. Ann Intern Med. 2015;162(9):641-650. doi:10.7326/ M15-0483

3. Åsvold BO, Vatten LJ, Midthjell K, Bjøro T. Serum TSH within the reference range as a predictor of future hypothyroidism and hyperthyroidism: 11-year follow-up of the HUNT Study in Norway. $J$ Clin Endocrinol Metab. 2012;97(1):93-99. doi:10.1210/jc.2011-1724

4. Inoue K, Tsujimoto T, Saito J, Sugiyama T. Association between serum thyrotropin levels and mortality among euthyroid adults in the United States. Thyroid. 2016;26(10):1457-1465. doi:10.1089/ thy. 2016.0156

5. Onat A, Aydin M, Can G, et al. Normal thyroid-stimulating hormone levels, autoimmune activation, and coronary heart disease risk. Endocrine. 2015;48(1):218-226. doi:10.1007/s12020-0140269-z

6. Fernández-Real JM, López-Bermejo A, Castro A, Casamitjana R, Ricart W. Thyroid function is intrinsically linked to insulin sensitivity and endothelium-dependent vasodilation in healthy euthyroid subjects. J Clin Endocrinol Metab. 2006;91(9):3337-3343. doi:10.1210/jc.20060841

7. Jun JE, Jin SM, Jee JH, et al. TSH increment and the risk of incident type 2 diabetes mellitus in euthyroid subjects. Endocrine. 2017;55 (3):944-953. doi:10.1007/s12020-016-1221-1

8. Ying H, Tang YP, Bao YR, et al. Maternal TSH level and TPOAb status in early pregnancy and their relationship to the risk of gestational diabetes mellitus. Endocrine. 2016;54(3):742-750. doi:10.1007/ s12020-016-1022-6

9. Bellastella G, Maiorino MI, Scappaticcio L, et al. TSH oscillations in young patients with type 1 diabetes may be due to glycemic variability. $J$ Endocrinol Invest. 2018;41(4):389-393. doi:10.1007/s40618-0170752-5
10. de Vries TI, Kappelle LJ, van der Graaf Y, et al. Thyroid-stimulating hormone levels in the normal range and incident type 2 diabetes mellitus. Acta Diabetol. 2019;56(4):431-440. doi:10.1007/s00592018-1231-y

11. Lu J, Wang L, Li M, et al. Metabolic syndrome among adults in China: the 2010 China noncommunicable disease surveillance. J Clin Endocrinol Metab. 2017;102(2):507-515. doi:10.1210/jc.2016-2477

12. Deng W, Li C, Zhang Y, et al. Serum C1q/TNF-related protein-3 (CTRP3) levels are decreased in obesity and hypertension and are negatively correlated with parameters of insulin resistance. Diabetol Metab Syndr. 2015;7:33. doi:10.1186/s13098-015-0029-0

13. Teng W, Shan Z, Teng X, et al. Effect of iodine intake on thyroid diseases in China. $N$ Engl $J$ Med. 2006;354(26):2783-2793. doi:10.1056/NEJMoa054022

14. Fekete C, Lechan RM. Central regulation of hypothalamic-pituitarythyroid axis under physiological and pathophysiological conditions. Endocr Rev. 2014;35(2):159-194. doi:10.1210/er.2013-1087

15. Ren Z, He M, Deng F, et al. Treatment of pretibial myxedema with intralesional immunomodulating therapy. Ther Clin Risk Manag. 2017;13:1189-1194. doi:10.2147/TCRM.S143711

16. Jølving LR, Nielsen J, Kesmodel US, Nielsen RG, Nørgård BM, Beck-Nielsen SS. Chronic diseases in the children of women with maternal thyroid dysfunction: a nationwide cohort study. Clin Epidemiol. 2018;10:1381-1390. doi:10.2147/CLEP.S167128

17. Chang CH, Yeh YC, Caffrey JL, Shih SR, Chuang LM, Tu YK. Metabolic syndrome is associated with an increased incidence of subclinical hypothyroidism - A Cohort Study. Sci Rep. 2017;7 (1):6754. doi:10.1038/s41598-017-07004-2

18. Ye Y, Xie H, Zeng Y, Zhao X, Tian Z, Zhang S. Association between subclinical hypothyroidism and blood pressure-a metaanalysis of observational studies. Endocr Pract. 2014;20(2):150158. doi:10.4158/EP13237.OR

19. He W, Li S, Wang B, et al. Dose-response relationship between thyroid stimulating hormone and hypertension risk in euthyroid individuals. $J$ Hypertens. 2019;37(1):144-153. doi:10.1097/HJH.0000000000001826

20. Solini A, Monzani F. Hypothyroidism and intermediate metabolism: a complex relationship. Thyroid. 2010;20(8):837-839. doi:10.1089/ thy. 2010.1652

21. Zhao M, Tang X, Yang T, et al. Lipotoxicity, a potential risk factor for the increasing prevalence of subclinical hypothyroidism? J Clin Endocrinol Metab. 2015;100(5):1887-1894. doi:10.1210/jc.2014-3987

22. Wang F, Tan Y, Wang C, et al. Thyroid-stimulating hormone levels within the reference range are associated with serum lipid profiles independent of thyroid hormones. J Clin Endocrinol Metab. 2012;97 (8):2724-2731. doi:10.1210/jc.2012-1133

23. Ahi S, Amouzegar A, Gharibzadeh S, et al. The association between normal range TSH and lipid profile. Horm Metab Res. 2017;49 (6):424-429. doi:10.1055/s-0043-110142

24. Yan YR, Liu Y, Huang H, et al. Iodine nutrition and thyroid diseases in Chengdu, China: an epidemiological study. QJM. 2015;108 (5):379-385. doi:10.1093/qjmed/hcu216

25. Zhai X, Zhang L, Chen L, et al. An age-specific serum thyrotropin reference range for the diagnosis of thyroid diseases in older adults: a cross-sectional survey in China. Thyroid. 2018;28:1571-1579. [Epub ahead of print]. doi:10.1089/thy.2017.0715

26. Jeon MJ, Kim WG, Kwon H, et al. Excessive iodine intake and thyrotropin reference interval: data from the Korean National Health and Nutrition Examination Survey. Thyroid. 2017;27(7):967972. doi: $10.1089 /$ thy. 2017.0078

27. Shan Z, Chen L, Lian X, et al. Iodine status and prevalence of thyroid disorders after introduction of mandatory universal salt iodization for 16 years in China: a cross-sectional study in 10 cities. Thyroid. 2016;26(8):1125-1130. doi:10.1089/thy.2015.0613

28. Lim JA, Kim HJ, Ahn HY, et al. Influence of thyroid dysfunction on serum levels of angiopoietin-like protein 6. Metabolism. 2015;64 (10):1279-1283. doi:10.1016/j.metabol.2015.06.015 
29. Sakurai M, Nakamura K, Miura K, et al. Association between a serum thyroid-stimulating hormone concentration within the normal range and indices of obesity in Japanese men and women. Intern Med. 2014;53(7):669-674. doi:10.2169/internalmedicine.53.1387

30. Garin MC, Arnold AM, Lee JS, Tracy RP, Cappola AR. Subclinical hypothyroidism, weight change, and body composition in the elderly: the Cardiovascular Health Study. J Clin Endocrinol Metab. 2014;99 (4):1220-1226. doi:10.1210/jc.2013-3591
31. Møllehave LT, Linneberg A, Skaaby T, Knudsen N, Jørgensen T, Thuesen BH. Trends in treatments of thyroid disease following iodine fortification in Denmark: a nationwide register-based study. Clin Epidemiol. 2018;10:763-770. doi:10.2147/CLEP.S164824

32. Chaker L, Cappola AR, Mooijaart SP, Peeters RP. Clinical aspects of thyroid function during ageing. Lancet Diabetes Endocrinol. 2018;6(9):733-742. doi:10.1016/S2213-8587(18)30028-7

Diabetes, Metabolic Syndrome and Obesity: Targets and Therapy

\section{Publish your work in this journal}

Diabetes, Metabolic Syndrome and Obesity: Targets and Therapy is an international, peer-reviewed open-access journal committed to the rapid publication of the latest laboratory and clinical findings in the fields of diabetes, metabolic syndrome and obesity research. Original research, review, case reports, hypothesis formation, expert opinion and commentaries are all considered for publication. The manuscript management system is completely online and includes a very quick and fair peer-review system, which is all easy to use. Visit http://www.dovepress.com/testimonials.php to read real quotes from published authors. 